\title{
XX. On certain types of electric discharge
}

\author{
Prof. D.N. Mallik F.R.S.E. \& Prof. A.B. Das M.Sc.
}

To cite this article: Prof. D.N. Mallik F.R.S.E. \& Prof. A.B. Das M.Sc. (1922) XX. On certain types of electric discharge, Philosophical Magazine Series 6, 43:253, 216-222, DOI: 10.1080/14786442208633865

To link to this article: http://dx.doi.org/10.1080/14786442208633865

$$
\text { 曲 Published online: } 08 \text { Apr } 2009 .
$$

Submit your article to this journal

Џلl Article views: 2

Q View related articles $\sqsubset$ 


\section{[ 216$]$}

XX. On Certain Types of Electric Discharge. By Prof.

D. N. Maluix, F.R.S.E., and Prof. A. B. DAS, M.Sc.*

1. T previous papers (Phil. Mag. Oct. 1908, Oct. 1912, 1 July 1916) we studied the behaviour of electric discharge in a De La Rive tube, under gradually decreasing. pressure. In the present series of experiments, tubes of ordinary pattern (with electrodes consisting of thin rods) were used, in order to verify the various theoretical deductions, previously arrived at.

2. Three tubes $\left(\mathrm{T}_{1}=34.4 \mathrm{~cm}\right.$. in length, $\mathrm{T}_{2}=14.5 \mathrm{~cm}$., and $\mathrm{T}_{3}=4.2 \mathrm{~cm}$.) were used in parallel, in order that the effects of length and. decreased pressure on the character of the discharge should be clearly observable. The induction-coil used gave a spark-length of $19.94 \mathrm{~cm}$. in air, with a spherical electrode of $3 \mathrm{~cm}$. in diameter.

3. At a pressure of $73 \mathrm{~mm}$. no discharge passes in $T_{1}$, it is spindle-shaped in $\mathrm{T}_{2}$ while in $\mathrm{T}_{3}$ it is in the form of a band. As the pressure is further reduced at $20.5 \mathrm{~mm}$. the discharge in $\mathrm{T}_{1}$ is spindle-shaped, $\mathrm{T}_{2}$ shows a band which was the stage reached in $\mathrm{T}_{3}$ at the higher pressure, while in $\mathrm{T}_{\mathbf{3}}$ the band is changing into a glow discharge.

4. On the theory previously worked out, the spindle-shape is due to the mutual repnlsion between the various streams of discharge, which is seen to be operative, in spite of the symmetry of the electrodes.

5. Moreover, during the band stage, according to theory previously worked out, the result of ionization is annulled by recombination. Now ionization depends on $\frac{\mathrm{X}}{p}$, where $\mathrm{X}$ is the electric intensity along the discharge and $p$ the pressure. This quantity, therefore, depends on $\frac{1}{l_{p}}$ where $l$ is the length of the tube. The effect of decreasing the length must, therefore, correspond to that of increasing the pressure. As, however, the potential difference itselt depends on length and pressure (arts. 15 and 16), there is not as yet sufficient material for working out the law of this correspondence.

6. It is found that it is only when the discharge is in the form of a thin band or a single stream of discharge, that there is an action (attraction or repulsion) when the dischargetube is placed in a transverse magnetic field. In the actual experiment, a rectangular coil of wire, carrying current, two

* Communicated by the Authors. 
of whose sides are parallel to the tube, was used to produce such a field, fig. 1 .

Fig. 1.

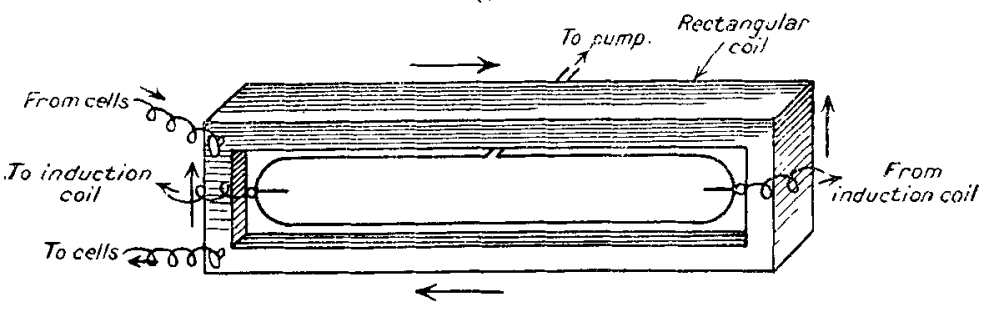

7. According to the theory previously worked out, this usual electrorlynamic action between currents will be observable only when the discharge is in the form of a band, the number of corpuscles in this case being equal to that of positive ions.

The present experiment, therefore, is in confirmation of this theory.

8. When the pressure is further reduced $\left(.03 \mathrm{~mm}\right.$.) $\mathrm{T}_{1}$ shows fine striatory discharge.

This tube thus does not at all show the band stage-which alone is affected by a magnetic field-with the inductioncoil used in the above experiment.

9. At this pressure, the discharge in $\mathrm{T}_{2}$ is striatory but the striæ are thicker ; while in $\mathrm{T}_{3}$ the positive column is entirely absent, the cathode glow extending over the whole length of the tube.

10. It will be seen that the characteristic changes in these discharge-tubes and the characteristic differences between the three tubes arising from a difference in length correspond to the peculiarities exhibited by the curves connecting pressure and potential difference in them.

Curve II, fig. 2, gives the relation between pressure and potential difference in $\mathrm{T}_{2}$.

11. In $\mathrm{T}_{2}$, at high pressure down to nearly $55 \mathrm{~cm}$., the showery stage is indicated by the curved line. From this pressure to nearly $25 \mathrm{~cm}$., the discharge is in the form of a band. This is indicated by a straight portion in the curve. The discharge behaves during this stage as an ordinary flexible wire carrying current. As the pressure is further reduced, the curve bends a way from the line of pressure, the difference of potential increasing enormously with decreased pressure, when the pressure is lowered below $2 \mathrm{~mm}$.

In the case of $T_{3}$, the first stage is absent, as well as the first portion of the curve. (Curve I, fig. 2.) 
In $\mathrm{T}_{1}$ the second stage is absent, and the second portion of the curve with it. (Curve III, fig. 2.)

Fig. 2.

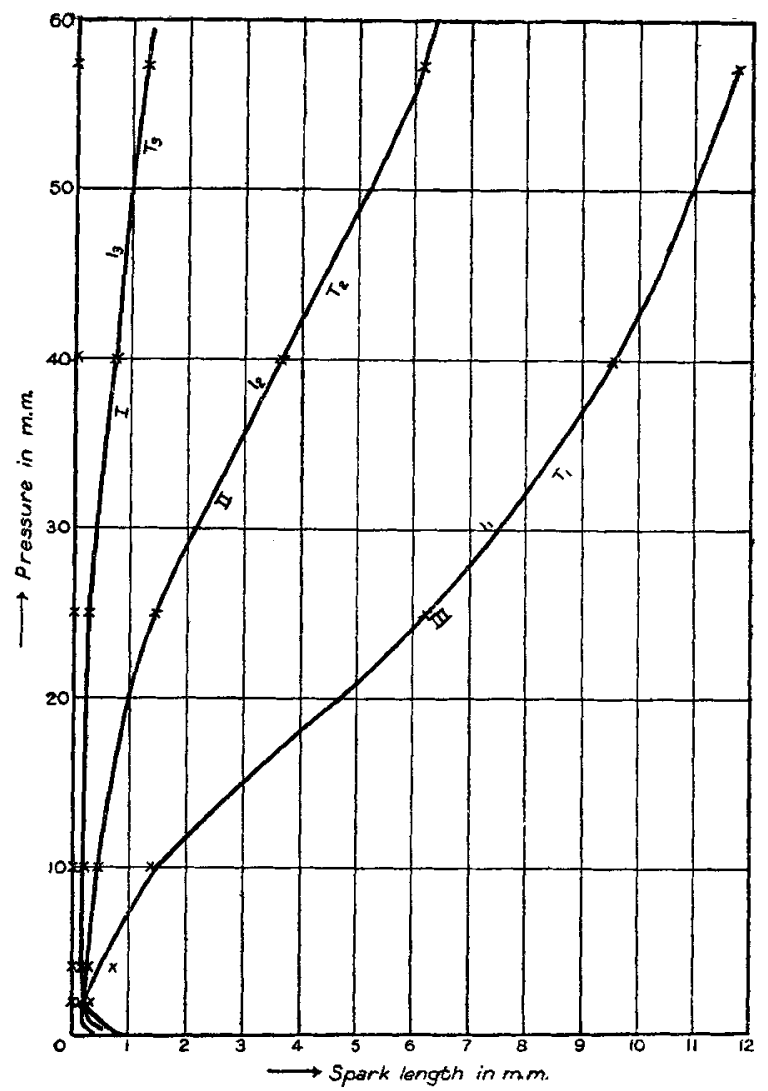

Spark-length of the induction-coil $=19.94 \mathrm{rm}$.

12. When, however, the voltage of the induction-coil is increased, the discharge in $\mathrm{T}_{1}$ also passes through all the various stages and the curve $I$ (fig. 3) exhibits the usual peculiarities in their entirety.

13. These characteristics are the same as those exhibited by De La Rive tubes. They are, therefore, common to ali electric discharges, as they should be on theoretical grounds. 14. It is possible to work out an approximate theory giving a relation between pressure, potential difference, and the length of the discharge-tube, but the formula 
obtained is much too complicated for discussion to serve any useful purpose, at the present stage.

Fig. 3.

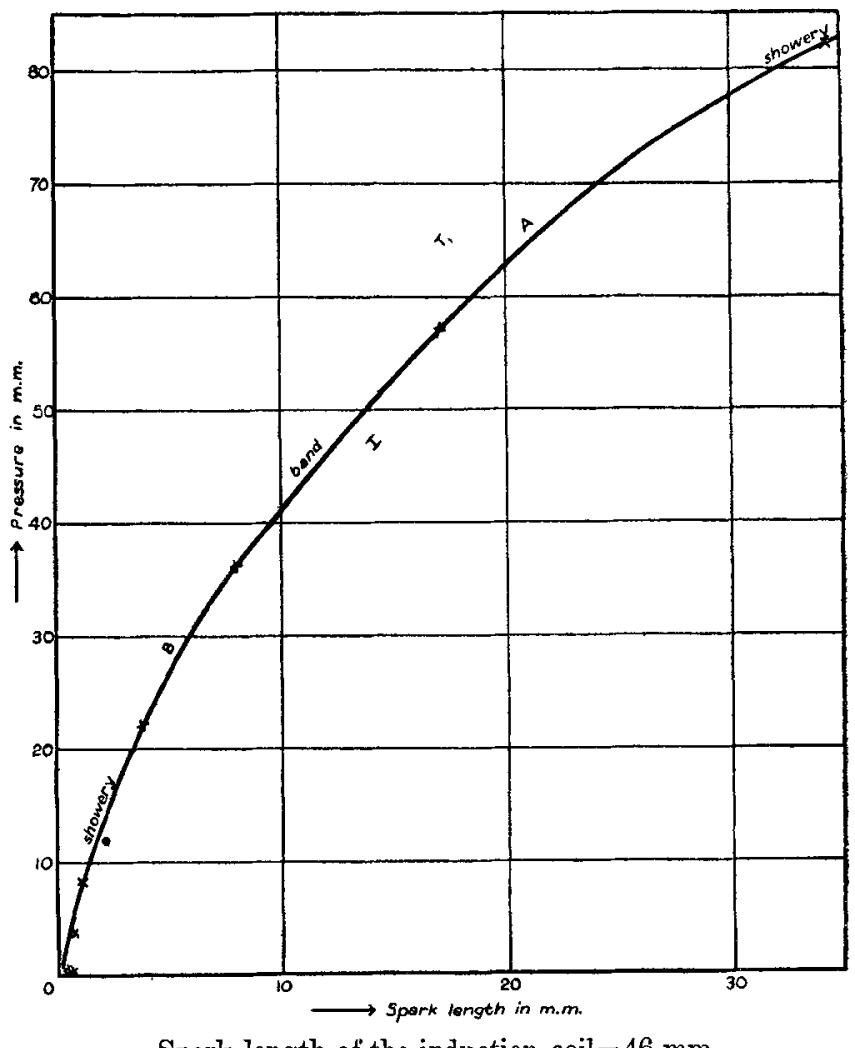

Spark-length of the induction-coil $=46 \mathrm{~mm}$.

15. In the particular case in which the curve is a straight line, the formula obtained in a previous paper was

$$
\mathrm{V}_{0}=\frac{\mathrm{X}}{p} \cdot \frac{q+q^{\prime} \frac{\lambda^{\prime}}{\lambda}}{q+q^{\prime}},
$$

where $V_{0}$ is the difference of potential between the terminals of the induction-coil when the circuit is open, $\mathrm{X}^{\prime}$ the electric intensity, assumed to be constant throughout the tube, $q, q^{\prime}$, velocities of + ions and corpuscles, and $\lambda, \lambda^{\prime}$, their mean free paths, $p$ being the pressure.

16. Now experiment shows that $\mathrm{X}$ itself depends on the 
discharge circuit, at any rate with an ordinary inductioncoil provided with a metallic interrupter, which is controlled by a spring. Thus, with such an induction-coil of sparklength in air equal to about $20 \mathrm{~mm}$., when the discharge was passed through the tube $T_{1}$, the interrupter worked with much greater frequency than when the tube $\mathrm{T}_{3}$ was in circuit. This is verified, by the curves $I$ and II, fig. 4.

Fig. 4.

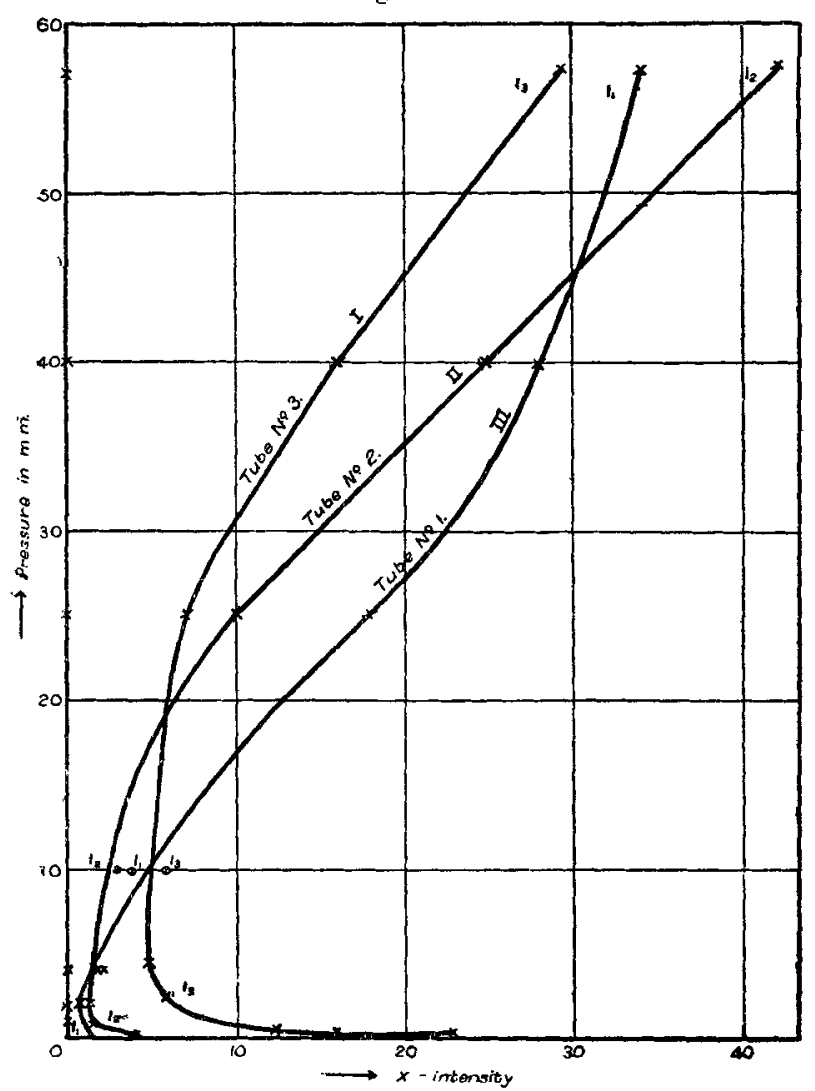

$\mathrm{X}=f(p)$. If in the above formula $\mathrm{X}$ were independent of the discharge-tube, the straight portions of the two curves ought to have been nearly coincident, if, at any rate, we could admit $q \propto \frac{1}{l}, q^{\prime} \propto \frac{1}{l}, \lambda \propto \frac{1}{l}, \lambda^{\prime} \propto \frac{1}{l}$.

17. The interdependence of the various factors that enter into the phenomena is clearly brought out by the curves 1 , 
2, 3, 4 (6g. 5). Thus, it is seen that for the same E.M.F. in the primary, the current as well as the character of the discharge depend on the pressure.

There is one feature of this phenomenon brought out by these curves, which is worthy of notice. When the discharge is in the form of a band (curve 1, fig. 5) (pressure $24 \mathrm{~mm}$.).

Fig. 5.

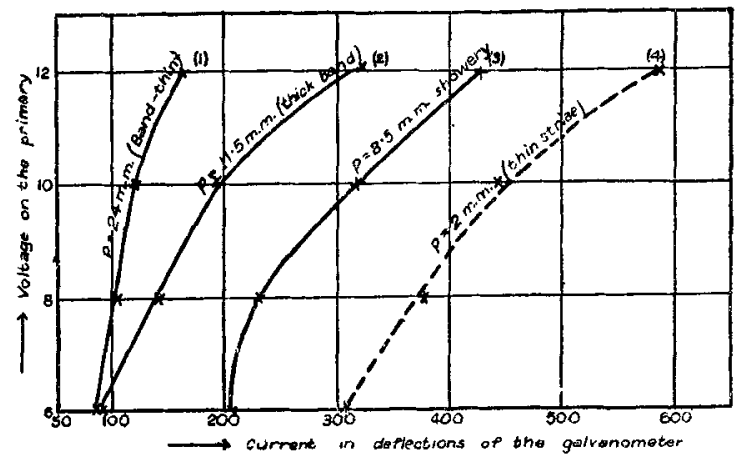

within a certain range, the current is proportional to the F.M.F. of the primary. Now, according to Ohm's law, the current in a wire circuit is proportional to the E.M.F. when the circuit is open. As we may reasonably take the E.M.F'. of the secondary, when no discharge passes, proportional to the E.M.F. in the primary, we conclude that the band discharge satisfies the criterion of a current in a wire circuit, in respect of Ohm's law. This is of interest in view of art. 6 .

18. When the striatory discharge (in tube $\mathrm{T}_{2}$ ) is placed in the centre of a rectangular coil carrying current, the number of striæ increases, and in the actual experiment they become inclined to their original directions, while as the current is reversed, the inclination is reversed also.

19. A tentative theory of these experimental results (art. 18) may be given as follows :-

The Faraday dark space is, as we have seen ("Electric Discharge in a Transverse Magnetic Field," Philosophical Magazine, July 1916), a region practically devoid of ions. If this is so, all the dark spaces in the striatory column must be held to be also regions containing but few ions, and per contra, the illuminated portions to mark regions where there is copious ionization.

20. A corpuscle being thrown off from the negative electrodes will be in a condition to ionize the gas almost directly, if the pressure of the gas in the tube is sufficiently 
high ; for the electric intensity near the cathode, in this case, being great, the energy required by a corpuscle for ionizing the gas will be acquired at once.

21. As the pressure decreases, the electric intensity near the cathode decreases also, and the corpuscle has to move through a finite distance under the action of the electric force before it acquires sufficient energy for producing ionization. The space through which a corpuscle shot off from the cathode has to traverse, before it is in a condition to ionize the gas, would be the Faraday dark space. The energy so acquired, howerer, is used ap in prolucing ionization in the first illuminated area. A corpuscle issuing from this illuminated area has, therefore, to move through some distance before it is again in a condition to ionize the gas. But as the average electric force at the strix is greater than along the Faraday dark space (H. A. Wilson, Proc. Camb. Phil. Soc. xi.) a corpusele has to move through a much shorter distance than the Faraday dark space, in order to recover the minimum energy required (Phil. Mag. Feb. 1920) for ionization. Accordingly, the dark space along the strice is of shorter length than the Faraday dark space.

22. When a radial magnetic field is introduced, a corpuscle issuing from the cathode tends to move under the electric force along a line of discharge and undergoes at the same time an angular displacement under the manetic force. The resultant velocity acquired attains to the minimum value required for ionization at a shorter distance from the cathode than when the magnetic field was not on. The effect of a magnetic field is, therefore, to shorten the Faraday dark space xnd all the other dark spaces and, pari passu, to increase the number of strice. Moreover, the ions in the striatory discharge, under the joint effect of electric and magnetic fields, move in a spiral, the tangent to the spiral at any point being inclined to the axis of the tube, one way or the other, according to the direction of the magnetic lines of torce in the field. Remembering that the movement of ions under the electric field itself produces a magnetic field inecessarily comparatively weak in comparison with the extraneous field that may be introduced), we conclude that ions in a striatory discharge in general move in spirals, more or less inclined to the axis of the tube, even without the application of a magnetic field.

Measurements are being undertaken for a quantitative verification of the above theory.

Our thanks are due to the authorities of the Presidency College, Calcutta, for facilities for carrying on our work there. 